\title{
Quantitative characterization of field emission parameters: Application to statistical analysis of individual carbon nanotubes/nanofibers
}

\author{
Florian Andrianiazy, Jean-Paul Mazellier, ${ }^{\text {a) }}$ Lucie Sabaut, Laurent Gangloff, and \\ Pierre Legagneux \\ Nanocarb Laboratory, Thales-Ecole Polytechnique, Route Départementale 128, 91767 Palaiseau Cedex, \\ France \\ Oliver Gröning \\ EMPA, Swiss Federal Laboratories for Materials Science and Technology, Überlandstrasse 129, \\ CH-8600 Dübendorf, Switzerland
}

(Received 11 August 2014; accepted 5 November 2014; published 19 November 2014)

\begin{abstract}
In this study, the authors present a detailed procedure for the quantitative measurement of the field emission properties of a large number of vertically aligned carbon nanotubes/nanofibers (CNs) using a scanning anode field emission microscope (SAFEM). This method provides the statistical distribution of all the relevant emitter parameters such as field enhancement factor, emitter height, and maximal current before failure. In order to extract the correct absolute enhancement factor of each CN, an analytical electrostatics model has been developed taking into account for the "tip-totip" geometry of anode and cathode in the SAFEM set-up. This analytical model has been validated by finite elements electrostatic simulations. Experimental measurements of enhancement factor distributions determined at several anode-cathode distances show the importance of this procedure to obtain quantitative correct values. A good correlation between the enhancement factor and the $\mathrm{CN}$ length has been observed. Additionally, the correlation between the maximum current before failure and the enhancement factor has also been investigated. Unlike in previously reported experiments, no clear dependence between these two parameters has been obtained. This result can be explained in our case by a large dispersion of CN crystalline quality or CN-substrate electrical contact resistance in the array used in this study. (C) 2014 American Vacuum Society.

[http://dx.doi.org/10.1116/1.4902019]
\end{abstract}

\section{INTRODUCTION}

Cold field emitters have been widely studied in the context of replacing thermionic sources in vacuum electronics components such as displays, ${ }^{1}$ traveling wave tubes ${ }^{2}$ (TWT), or X-ray sources. ${ }^{3}$ The requirement toward the cathode current density specifications for these applications ranges from 0.01 to $10 \mathrm{~A} / \mathrm{cm}^{2}$ where the current density is defined as the total emission current divided by the effective cathode area. One common feature for these cold emitters is the requirement for an electrical conductive material and a high geometrical aspect ratio to benefit from the field enhancing tip effect. In this respect, various microtips ${ }^{4,5}$ (molybdenum, tungsten, silicon, diamond, etc.), nanowires ${ }^{6,7}$ (silicon, zinc oxide, etc.), and carbon nanotubes/nanofibers ${ }^{8}$ (CNs) have been intensely studied. CNs are regarded as very good candidates as they present exceptional electrical, thermal, and mechanical properties and can exhibit very high aspect ratio. The large disproportion between tip emitting surface and effective cathode surface requires to operate in parallel many individual emitters, each one delivering a significant current, in order to obtain useful cathode current densities. Whereas large ${ }^{9}$ and stable ${ }^{10}$ currents from individual CNs have been already demonstrated, achieving high current densities in $\mathrm{CN}$ arrays is challenging.

Field emission obeys the Fowler-Nordheim (FN) equation ${ }^{11}$ with an exponential dependence of the emitted current

${ }^{\text {a)} E l e c t r o n i c ~ m a i l: ~ j e a n-p a u l . m a z e l l i e r @ t h a l e s g r o u p . c o m ~}$ on the local field at the $\mathrm{CN}$ apex. Operating many emitters in parallel imposes that these emitters exhibit a similar aspect ratio, i.e., a narrow distribution of the field enhancement factor. Arrays of vertically aligned CNs are particularly promising because uniform $\mathrm{CNs}$ can be grown ${ }^{12}$ and the field screening effect between neighboring CNs is also uniform. ${ }^{13}$

Global characterizations, such as obtained from I(V) characteristics in a diode configuration, give the collective behavior of such sources, which are often difficult to relate to the microscopic structure of the cathode. However, variations of geometrical and physical properties are expected in vertically aligned $\mathrm{CN}$ arrays, affecting the performances of the cathode. The evaluation of these variations requires measuring the emission from individual emitters. Very few experiments have been focused on this particular aspect ${ }^{10,14}$ and each required hand-tuning of the setup to ensure a good measurement. This limited the analysis to a very little number of emitters because of the time consumption of the measurement. A more refined measurement of the single emitter properties and their variation can be performed in a scanning anode field emission microscope (SAFEM) $\operatorname{setup}^{9,13}$ (Fig. 1). This method allowed scanning for large areas (and large number of emitters as a consequence) for field emission. Nevertheless, the treatment of the electrostatics of the SAFEM system lacked for a real description of the anode to cathode equipotential distribution.

Based on an electrostatic model, we present here a SAFEM measurement procedure allowing us to evaluate the absolute enhancement factor of CNs $\left(\beta_{0}\right)$ and their maximum 


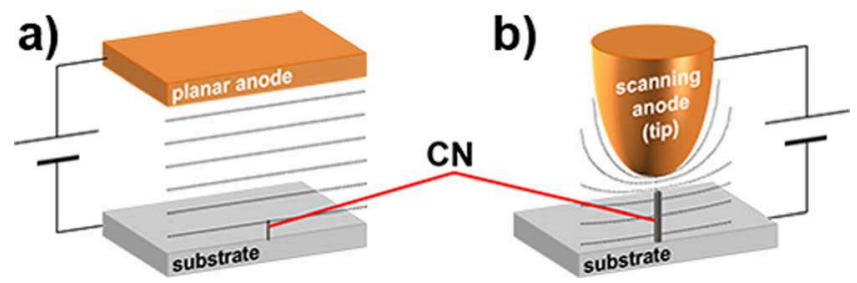

FIG. 1. (Color online) (a) Planar cathode-anode configuration. (b) Scanning anode field emission microscope configuration. In both configurations, the shape of the equipotentials does not take into account the tip effect introduced by the presence of the $\mathrm{CN}$ (whom phantom representation is given for reader convenience).

emitted currents $\left(i_{\max }\right)$. To the best of our knowledge, this maximum emitted current distribution, which is a critical factor in limiting the cathode current density, has never accurately been studied. This study, which provides the distribution of $i_{\max }$ and $\beta_{0}$, gives a better understanding of the factors limiting the current density delivered by vertically aligned $\mathrm{CN}$ arrays.

\section{CN ELECTROSTATIC MODEL}

Field emission of electrons from a metallic surface is enabled by quantum mechanical tunneling under the action of a strong external electric field. The relationship between $\mathrm{CN}$ emitted current $i_{\mathrm{CNT}}$ and the electric field at $\mathrm{CN}$ apex $E_{\text {apex }}$ is described by the $\mathrm{FN}$ equation ${ }^{11}$

$$
i_{\mathrm{CNT}}=A E_{\text {apex }}^{2} \exp \left(-\frac{B}{E_{\text {apex }}}\right),
$$

where $A$ and $B$ are physical constants, which depend on the work function $\Phi$ of $\mathrm{CNs}$, where the FN elliptical functions are taken to be unity. ${ }^{15}$ In this study, we assumed ${ }^{16-18} \Phi=4.9 \mathrm{eV}$. Under this assumption, we derived values of $A=1.25$ $\times 10^{-19} \mathrm{~A} \mathrm{~m}^{2} \mathrm{~V}^{-2}$ (for a $\mathrm{CN}$ radius equal to $25 \mathrm{~nm}$ ) and $B=7.12 \times 10^{10} \mathrm{~V} \mathrm{~m}^{-1}$, which are used throughout this paper.

\section{A. Planar configuration}

The $\mathrm{CN}$ in a field emission set-up is part of an electrostatic configuration containing different electrodes $(\mathrm{CN}$ itself is an electrode of the system) with nontrivial geometry. The electric field at $\mathrm{CN}$ apex can be derived from this electrostatic system by solving the zero-charge Poisson equation in the vacuum region (we consider no space charge here). The most commonly considered system is the planar diode anode-cathode configuration, where the anode-cathode separation is usually much larger than the emitter height [Fig. 1(a)]. In this case, the usual practice is to define a uniform macroscopic field $E_{\text {ext }}$ (in the absence of $\mathrm{CN}$ emitters) equal to the anode-cathode voltage divided by the cathode to anode distance. A $\mathrm{CN}$ placed in this external macroscopic field experiences an electrical field at apex, $E_{\text {apex }}$, that can be written as

$$
E_{\text {apex }}=\beta_{0} E_{\text {ext }} \text {. }
$$

$\beta_{0}$ is the $\mathrm{CN}$ field enhancement factor. This factor describes the tip effect induced by the $\mathrm{CN}$ geometry. As CNs can exhibit high aspect ratio (length over radius), the electric field experienced at $\mathrm{CN}$ apex is much larger than the macroscopic external field. Thus, relatively low macroscopic electric fields (typically few $\mathrm{V} / \mu \mathrm{m}$ ) can induce at $\mathrm{CN}$ apex the large electric field (few $\mathrm{kV} / \mu \mathrm{m}$ ) required to induce significant field emission currents. This enhancement factor is thus a figure of merit for $\mathrm{CN}$ emitters and it has been widely studied. 9,13,19 An important question is how the enhancement factor can be calculated from parameters describing the emitter geometry and several models ${ }^{20-22}$ have been proposed for this. In an emitter array structure, the planar structure described in Fig. 1(a) generates a uniform macroscopic field in the absence of CN emitters: all CNs of a cathode experience current emission in parallel and a collective behavior is measured; however, it is impossible to access the behavior of the individual CNs.

\section{B. SAFEM configuration}

Accessing this individual emitter behavior and its statistical distribution in the array allows a more precise understanding of the cathode performances and the influence of different steps in its production (catalyst production, growth recipe, etc.). In contrast to the planar configuration, in SAFEM, the anode is a micrometric conductive tip placed at a few microns from the emitter structure under investigation. In the absence of the $\mathrm{CN}$ emitter, this configuration generates a nonuniform, locally confined electric field. This is a key feature allowing SAFEM configuration for probing $\mathrm{CNs}$ one by one.

This nonuniform electric field prevents from using Eq. (2) for a quantitative determination of the enhancement factor of the probed $\mathrm{CN}$. It has been realized that the assumption of a planar configuration, i.e., application of Eq. (2), in the SAFEM leads to a systematic underestimation of the enhancement factor; however, up to now, no model has been proposed accounting quantitatively for the nonuniform electric field. ${ }^{23}$ Application of Eq. (2) leads to major variations in extracting the enhancement factor of $\mathrm{CNs}$ with the cathode to tip anode distance. ${ }^{14,24}$

We developed an electrostatic model based on the calculation of the potential generated by the SAFEM tip in the absence of the $\mathrm{CN}$ emitter. The apex field on the $\mathrm{CN}$ emitter can then be derived assuming that $\mathrm{CN}$ induces a local perturbation of the potential field. This model, which takes the anode- $\mathrm{CN}$ distance into account, allows extracting quantitatively correct enhancement factors for $\mathrm{CN}$ populations.

Furthermore, our procedure gives a direct access to the maximum emitted current before failure distribution of our $\mathrm{CN}$ array allowing the investigation of a possible correlation between the maximum emitted current and the geometric characteristics of a $\mathrm{CN}$.

\section{Local electric field calculation}

In SAFEM configuration [Fig. 1(b)], the electric field between the planar substrate and the SAFEM tip is not uniform as schematically represented in Fig. 2.

For our analytical model, we assume a perfectly conductive tip (representing the scanning anode tip) with axial symmetry pointing perpendicular to a grounded planar electrode 


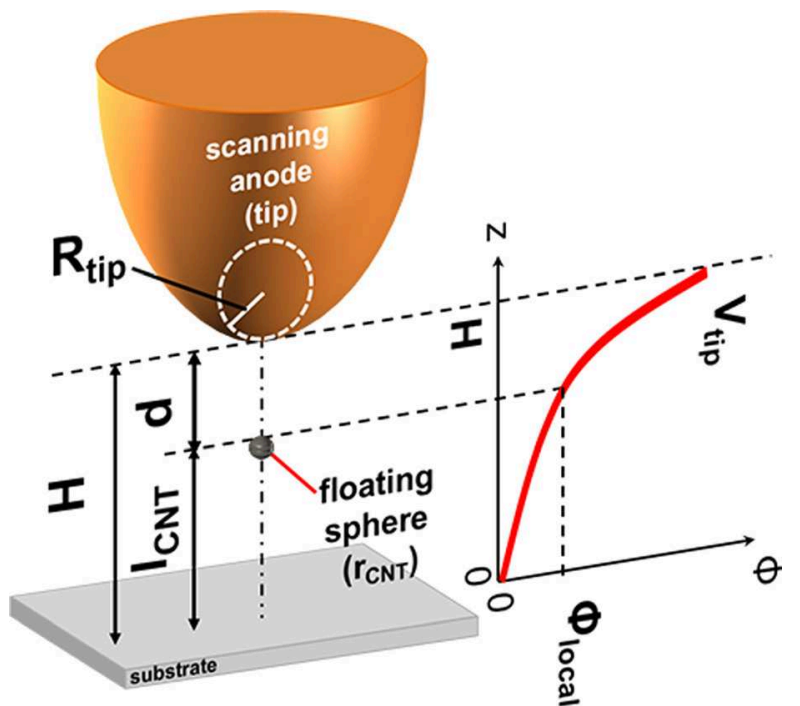

FIG. 2. (Color online) Hyperbolic-floating sphere model. The CN-tip distance is denoted as $d$, the tip-substrate distance is denoted as $H$. Schematic representation of the electrical potential along symmetry axis is also given (in the absence of the floating sphere).

(representing the cathode substrate). We take this last surface as the reference for height measurement. The planar substrate potential is defined as potential reference. We assume the tip to present a radius of curvature $R_{\text {tip }}$, biased at voltage $V_{\text {tip. }}$ The minimum distance between the tip surface and the grounded planar surface is denoted as $H$. We can write the electrical potential expression on the symmetry axis of the system as a function of height $z$ and $H$ as

$$
\phi_{\text {local }}(z, H)=V_{\text {tip }} \times \gamma_{R \text { tip }}(z, H) .
$$

If we assume the tip is represented by an hyperboloid surface (without loss of generality on our results), we can express $^{25}$ the dimensionless geometrical factor $\gamma_{\mathrm{Rtip}}(z, H)$ as

$$
\gamma_{R \text { tip }}(z, H)=\ln \left(\frac{1+\frac{z|\cos (\alpha)|}{H}}{1-\frac{z|\cos (\alpha)|}{H}}\right) \frac{1}{\ln \left(\frac{1+|\cos (\alpha)|}{1-|\cos (\alpha)|}\right)} \text {. }
$$

$\alpha$ being $\tan ^{-1}\left(\sqrt{R_{\mathrm{tip}} / H}\right)$. Note that for $z=H$, at tip surface, $\gamma_{R \text { tip }}(H, H)=1$, i.e., $V_{\text {local }}=V_{\text {tip }}$, and for $z=0$, at substrate surface, $\gamma_{R \text { tip }}(0, H)=0$, i.e., $V_{\text {local }}=0$.

Now we place on the symmetry axis, a conductive sphere of radius $r_{\mathrm{CN}}$, which represents a $\mathrm{CN}$, according to the floating sphere model. ${ }^{22}$ We assume the electrical potential of the floating sphere (which is virtually connected to the substrate, i.e., at reference potential) locally perturbing the potential of the SAFEM tip to planar cathode system. The floating sphere center is at height $l_{\mathrm{CNT}}$ and the sphere-tip distance is denoted as $d$. Note that the perturbation assumption is supported by finite element modeling simulation (not shown here) under the constraint that the sphere radius has to be significantly smaller than $H$ and $d$, constraints that are fulfilled in our practical case. Introducing this grounded sphere perturbs the system: charges will be generated on the sphere surface, resulting in an electric field that will fall off rapidly away from the sphere. The sphere geometry allows assuming an isotropic induced electric field. According to the Gauss law, the value of this electric field on the sphere surface, $E_{\text {apex }}$, is

$$
E_{\text {apex }}=-\frac{\phi_{\text {local }}\left(l_{\mathrm{CNT}, H}\right)}{r_{\mathrm{CNT}}} .
$$

Note that this approach can also be done in case of a planar geometry. In planar geometry, the potential $\phi_{\text {local }}(z, H)$ would evolve linearly leading to a simple relation $\phi_{\text {local }}=l_{\mathrm{CNT}} \times E_{\text {ext }}$. Thus, we can derive the enhancement factor as described in Eq. (2) as $\beta_{0}=l_{\mathrm{CNT}} / r_{\mathrm{CNT}}$. We recover the same result as Wang et al., ${ }^{22}$ which is another support to our approach.

Replacing $\phi_{\text {local }}$ by its expression of Eq. (3) in Eq. (5), we obtain for the SAFEM configuration

$$
E_{\text {apex }}=-\frac{\gamma_{R \text { tip }}\left(l_{\mathrm{CNT}}, H\right)}{r_{\mathrm{CNT}}} V_{\text {tip }} .
$$

One can note that the electric field on the sphere strongly depends on the anode tip radius. In contrast to the planar configuration, the potential does not evolve linearly as a function of $\mathrm{z}$. As a result, we cannot derive a simple relation between the external anode-cathode voltage (or field) and this local potential: the use of macroscopic field is not appropriate in this configuration. In fact, for any nonplanar configuration, the evaluation of the local potential is a prerequisite to compute the correct electric field at the emitter apex.

We compared our analytical model to finite elements modeling (FEM) using COMSOL MULTIPHYSICS software by simulating structures depicted in Fig. 2. In experiment, the radius of curvature of the scanning anode tip is typically comprised between 1 and $5 \mu \mathrm{m}$ based on scanning electron microscope (SEM) investigations. Here, we assumed a tip radius $R_{\text {tip }}=2 \mu \mathrm{m}$. We studied the effect of the sphere position $\left(l_{\mathrm{CNT}}\right)$ and tip to sphere distance.

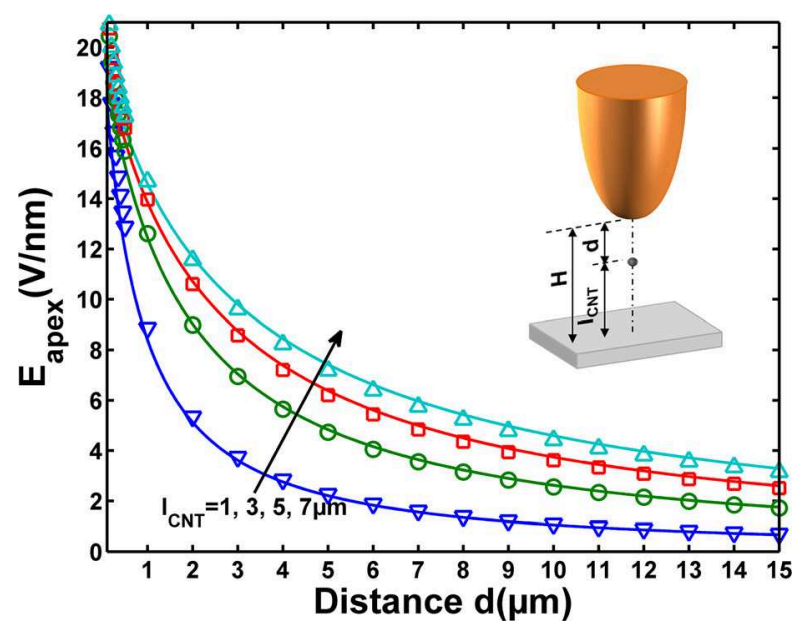

FIG. 3. (Color online) Comparison of calculated (lines) and simulated (markers) values of $\mathrm{E}_{\text {apex }}$ for four different floating sphere positions. $\mathrm{V}_{\text {tip }}$ bias is kept constant at $500 \mathrm{~V}$. 
The numerically simulated electric field on the sphere is directly compared to $E_{\text {apex }}$ calculated with the analytical model of Eq. (6), taking $V_{\text {tip }}=500 \mathrm{~V}$ and $r_{\mathrm{CNT}}=25 \mathrm{~nm}$. Figure 3 represents the calculated and simulated $E_{\text {apex }}$ as a function of the distance $d$ (sphere to tip distance).

We note a very good agreement between simulated and calculated values for distances above $500 \mathrm{~nm}$. The deviation observed for short distances is due to a coupling between the sphere and the tip beyond the perturbation approximation. The perturbation hypothesis is no more relevant, as expected because the required constraints, as defined previously, are not fulfilled. Despite, the excellent agreement between simulation and our model (in its validity domain) proves our approach to be correct.

In the case of a real $\mathrm{CN}$, a more precise evaluation of $E_{\text {apex }}$ is obtained by numerically simulating a hemisphere on a poststructure. Good agreement between the FEM simulation results and the analytical model can be obtained by introducing a proportionality factor of 0.7 in Eq. (6). Note that Edgecombe and Valdrè ${ }^{20}$ found the same factor in the case of a planar configuration. This correction factor is due to the presence of the conductive post. Thus, in the experimental part, we will consistently calculate the enhancement factor as $\beta_{0}=0.7\left(l_{\mathrm{CNT}} / r_{\mathrm{CNT}}\right)$. The comparison between the analytical model and the FEM simulations is shown in Fig. 4. Introducing this correction factor allows to extend our approach to more realistic $\mathrm{CN}$ geometry without loss of generality. In summary, at a distance above $2 \mu \mathrm{m}$, if one has access to the $\mathrm{CN}$ length and to the distance between the tip and the $\mathrm{CN}$, the value of $\gamma_{\text {Rtip }}\left(l_{\mathrm{CNT}}, H\right)$ can be evaluated using Eq. (5). For $\mathrm{CN}$ lengths between 3 and $7 \mu \mathrm{m}$, the error is below $5 \%$.

\section{Enhancement factor extraction}

Considering one $\mathrm{CN}$, knowledge of its length $l_{\mathrm{CNT}}$ allows to derive $\gamma_{R \text { tip }}\left(l_{\mathrm{CNT}}, H\right)$ directly from Eq. (5). By combining Eqs. (1) and (6), one can write

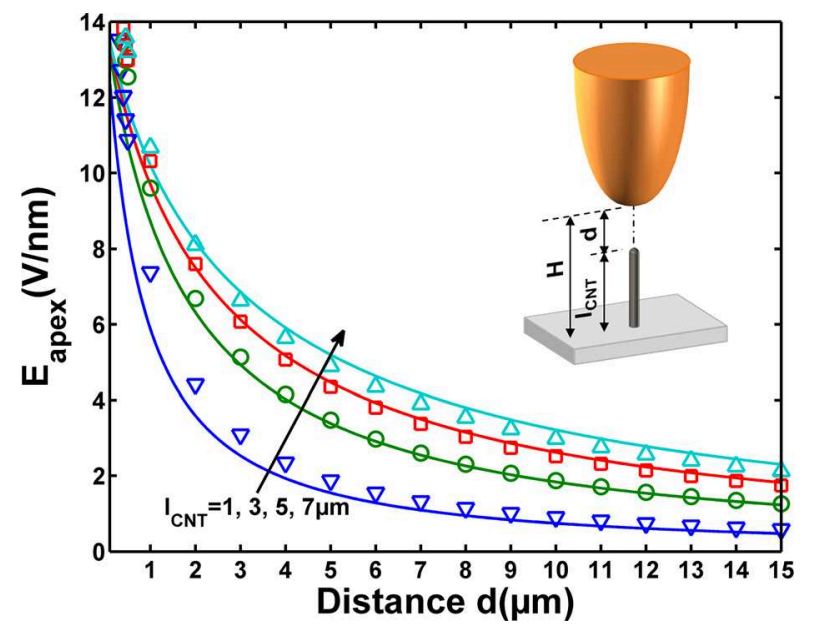

FIG. 4. (Color online) Comparison of calculated (lines) and simulated (markers) values of $\mathrm{E}_{\text {apex }}$ for four different $\mathrm{CN}$ lengths. $\mathrm{V}_{\text {tip }}$ bias is kept constant at $500 \mathrm{~V}$. Equation (6) has been multiplied by a factor 0.7 to fit the simulations.
TABLE I. Local electric field and enhancement factor expression for planar and SAFEM configurations.

\begin{tabular}{lcc}
\hline \hline & Planar configuration & SAFEM configuration \\
\hline Electric field $E_{\text {apex }}$ & $E_{\text {apex }}=-\frac{l_{\mathrm{CNT}}}{H} \frac{V_{\text {tip }}}{r_{\mathrm{CNT}}}$ & $E_{\text {apex }}=-\gamma_{R \text { tip }}\left(l_{\mathrm{CNT}}, H\right) \frac{V_{\text {tip }}}{r_{\mathrm{CNT}}}$ \\
$\begin{array}{l}\text { Enhancement factor } \\
\text { from the slope } \\
\text { of the F-N curve }\end{array}$ & $\beta_{0}=-\frac{B}{S} H$ & $\beta_{0}=-\frac{B}{S} \frac{l_{\mathrm{CNT}}}{\gamma_{R \text { tip }}\left(l_{\mathrm{CNT}}, H\right)}$ \\
\hline \hline
\end{tabular}

$$
\ln \left(\frac{I_{\mathrm{CNT}}}{V_{\text {tip }}^{2}}\right)=-\frac{B r_{\mathrm{CNT}}}{\gamma_{R \text { tip }}\left(l_{\mathrm{CNT}}, H\right) V_{\text {tip }}}+\ln (A) .
$$

The slope $S$ of the Fowler-Nordheim curve can be identified by plotting $\ln \left(I_{\mathrm{CNT}} / V_{\text {tip }}^{2}\right)$ vs $1 / V_{\text {tip }}$, as

$$
S=-\frac{B r_{\mathrm{CNT}}}{\gamma_{R \mathrm{tip}}\left(l_{\mathrm{CNT}}, H\right)} .
$$

Thus, we can calculate the corresponding radius of the emitter

$$
r_{\mathrm{CNT}}=-\frac{\gamma_{R \mathrm{tip}}\left(l_{\mathrm{CNT}}, H\right) S}{B} .
$$

Since we considered that the $\mathrm{CN}$ length is known, the geometric enhancement factor $\beta_{0}$ can be expressed as

$$
\beta_{0}=\frac{l_{\mathrm{CNT}}}{r_{\mathrm{CNT}}}=-\frac{B}{S} \frac{l_{\mathrm{CNT}}}{\gamma_{R \text { tip }}\left(l_{\mathrm{CNT}}, H\right)} .
$$

Table I summarizes the main differences between the classical approach and our new method.

If a planar configuration is assumed, the enhancement factor is under-estimated by a factor $\left(H / l_{\mathrm{CNT}}\right) \gamma_{R \text { tip }}(z, H)$. This is well confirmed by Bonard et al. ${ }^{14}$ for $1.4 \mu \mathrm{m}$ CNs and a $2.65 \mu \mathrm{m}$ tip-substrate distance where a factor 2 has been observed between calculated and experimental values. This value is close to the value calculated with our new method. Thus, the induced error is directly correlated to the distance between the $\mathrm{CN}$ and the anode tip. We will see in Sec. IV that this effect has been confirmed experimentally.

\section{EXPERIMENT}

A rectangular regularly spaced array of vertically aligned $\mathrm{CNs}$ has been grown by plasma-enhanced chemical vapor deposition at $700{ }^{\circ} \mathrm{C}$ for $20 \mathrm{~min}$ from nickel catalyst dots using an Aixtron Black Magic Reactor. For details of the process, refer to Teo et al. ${ }^{8}$ The mean length of the grown CNs measured by SEM is $5 \mu \mathrm{m}$.

The pitch between neighboring CNs is $40 \mu \mathrm{m}$ in order to insure individual $\mathrm{CN}$ characterization (negligible contribution from neighbor $\mathrm{CNs}$ during field emission measurements). This assumption is valid since the typical distance from CN to SAFEM tip is between 1 and $10 \mu \mathrm{m}$, in comparison to $40 \mu \mathrm{m}$ for first neighbors. If the network pitch is too small (e.g., $10 \mu \mathrm{m}$ ), the electric field at the apex of the neighboring CNs would not be negligible, thus allowing cross-talk 


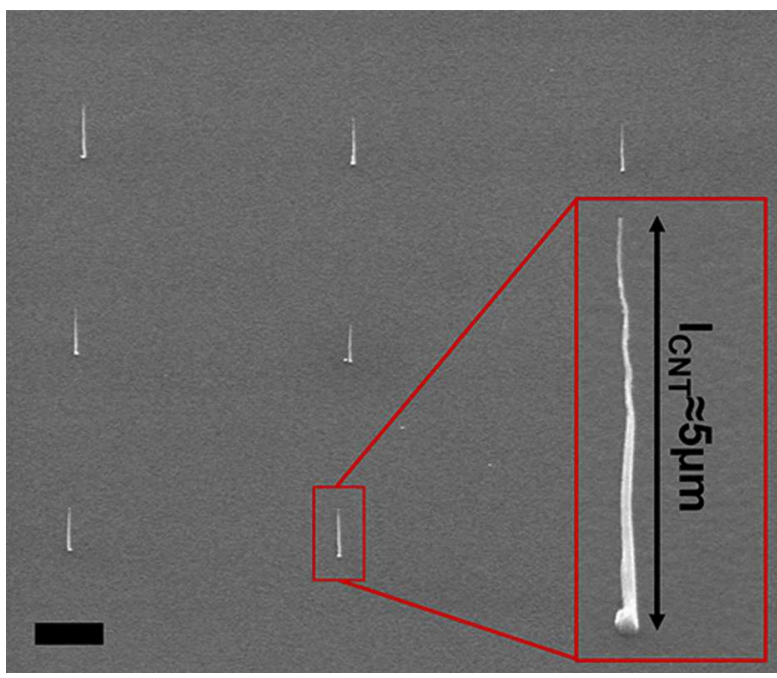

FIG. 5. (Color online) SEM image of a vertically aligned CN array (scalebar is $5 \mu \mathrm{m}$ ). Inset: zoom on one individual $\mathrm{CN}$ emitter of height $5 \mu \mathrm{m}$.

in field emission current. We measured the field emission characteristics of $300 \mathrm{CNs}$. Figure 5 shows a typical CN array characterized in the SAFEM.

The SAFEM anode tip is produced by electrochemical etching of a tungsten wire. Our process leads to tips with a radius of curvature between 1 and $5 \mu \mathrm{m}$ (Fig. 6).

\section{A. Tip radius of curvature determination}

On a unique $\mathrm{CN}$ previously observed by SEM (thus, its length $l_{\mathrm{CNT}}$ is known), we performed I-V characterizations at several CN-tip distances. For each distance $d$, we extract the slope $S(d)$ of the FN curve. Using Eq. (7), we can express $\gamma_{R \text { tip }}\left(l_{\mathrm{CNT}}, l_{\mathrm{CNT}}+d\right)$ as

$$
\gamma_{R \operatorname{tip}}\left(l_{\mathrm{CNT}}, l_{\mathrm{CNT}}+d\right)=\gamma_{R \operatorname{tip}}\left(l_{\mathrm{CNT}}, l_{\mathrm{CNT}}\right) \frac{S(0)}{S(d)}
$$

with $S(0)$ the value of the slope at $d=0$ (in practice, $d=100 \mathrm{~nm})$. Since $\gamma_{R \text { tip }}\left(l_{\mathrm{CNT}}, l_{\mathrm{CNT}}\right)=1$, then $\gamma_{R \text { tip }}\left(l_{\mathrm{CNT}}\right.$,

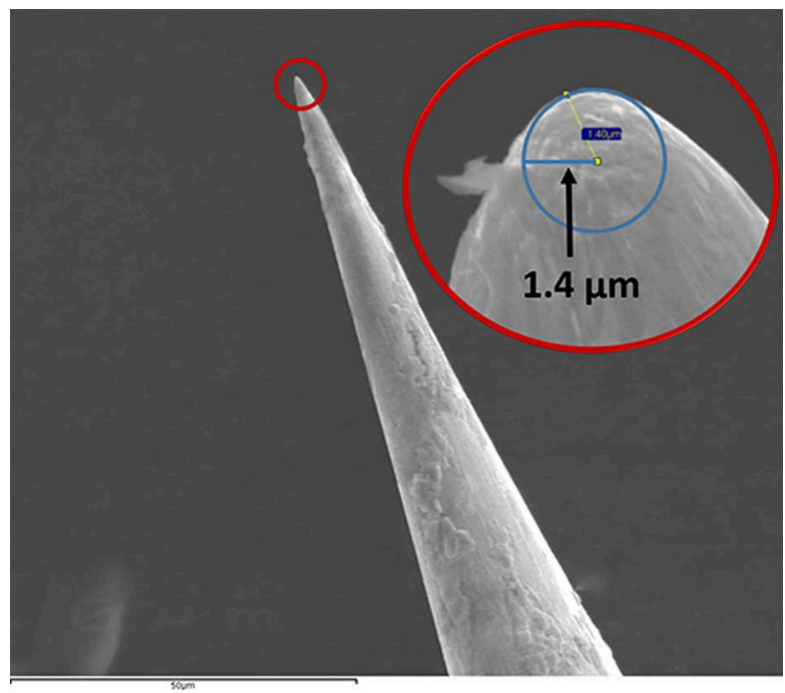

FIG. 6. (Color online) SEM image of a SAFEM tip made of tungsten wire. The evaluated radius of curvature is $1.4 \mu \mathrm{m}$.

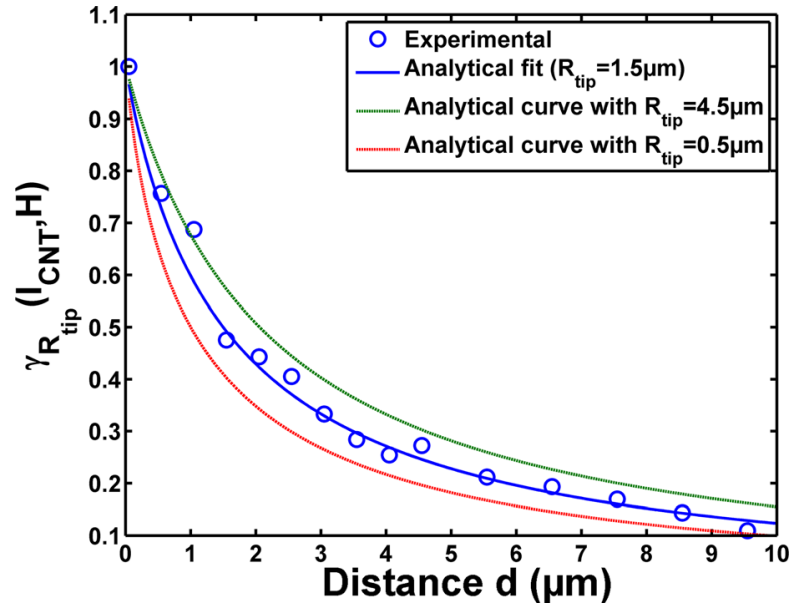

FIG. 7. (Color online) Experimental $\gamma_{R \text { tip }}\left(l_{\mathrm{CNT}}, H\right)$ as a function of anode tip to $\mathrm{CN}$ distance and analytical fit from Eq. (5). The analytical curves for $R_{\text {tip }}=0.5 \mu \mathrm{m}$ and $R_{\text {tip }}=4.5 \mu \mathrm{m}$ are also presented.

$\left.l_{\mathrm{CNT}}+d\right)=(S(0) / S(d))$. Thus, we have access to the experimental values of $\gamma_{R \text { tip }}\left(l_{\mathrm{CNT}}, l_{\mathrm{CNT}}+d\right)$. Using Eq. (5), by fitting the radius of curvature of the tip, which is the only unknown parameter in this case, the best optimal curve is determined (Fig. 7). In this case, we extract $R_{\text {tip }}=1.5 \mu \mathrm{m}$, which is in good agreement with the value found by SEM investigation of the tip (Fig. 6). Figure 7 also illustrates the analytical curves for $R_{\text {tip }}=$ $0.5 \mu \mathrm{m}$ and $R_{\text {tip }}=4.5 \mu \mathrm{m}$, and shows that the fit precision is satisfying regarding the sensibility of $\gamma_{R \text { tip }}\left(l_{\mathrm{CNT}}, H\right)$ with $R_{\text {tip }}$.

\section{B. Measurement procedure}

In order to automatically characterize a large number of CNs with regard to their field emission properties, the following procedure is implemented:

\section{Localization of the $\mathrm{CNs}$}

First, we have to precisely localize the lateral position each $\mathrm{CN}$ on the substrate. For this purpose, a large area of the substrate is scanned by the tip, kept at constant height, while sourcing a small constant current (here, $11 \mathrm{nA}$ ) and measuring the required extraction voltage. When the anode tip is positioned directly above an emitting $\mathrm{CN}$, the extraction voltage will show a minimum at this position. This modulation in the extraction voltage is mapped, revealing the $\mathrm{CN}$ array structure. Each minimum voltage point on the map represents a $\mathrm{CN}$ position. These points are automatically found by a MATLAB routine and kept in memory. Figure 8 shows a typical extraction voltage map $\mathrm{V}(\mathrm{x}, \mathrm{y})$ at constant height and constant emission current of $11 \mathrm{nA}$.

\section{Position of the apex of each $\mathrm{CN}$}

We have simulated a SAFEM tip approaching gradually a $\mathrm{CN}$ apex. From Eq. (1), we have simulated by FEM the required tip voltage, as a function of $d$, inducing a constant emission current from the single $\mathrm{CN}$ ( $11 \mathrm{nA}$ in this work). Our results are summarized on Fig. 9 for different $\mathrm{CN}$ lengths. At distances larger than $1 \mu \mathrm{m}$, the required voltage is nearly linearly dependent of the distance $d$. The slope is 


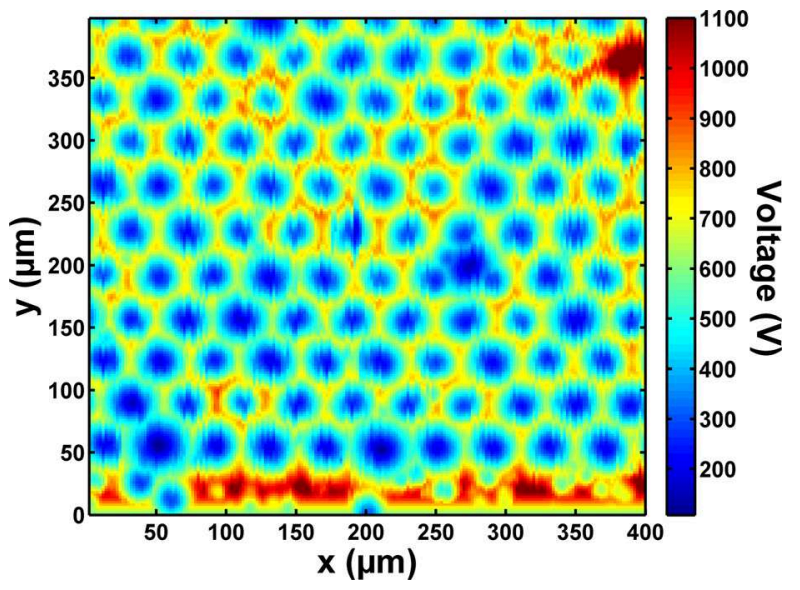

FIG. 8. (Color online) Example of a typical extraction voltage map at constant height and constant current of $11 \mathrm{nA}$.

highly dependent on the $\mathrm{CN}$ length, i.e., on the field enhancement factor. For distances $d$ below $1 \mu \mathrm{m}$, however, the voltage does not evolve linearly anymore and we observe an asymptotic behavior, independent on the $\mathrm{CN}$ length at a constant $\mathrm{CN}$ apex radius of $25 \mathrm{~nm}$. For a $\mathrm{CN}$-tip distance of $100 \mathrm{~nm}$, whatever the CN length is, a voltage of $90 \mathrm{~V}$ is required to extract $11 \mathrm{nA}$ from CNs. Thus, on each $\mathrm{CN}$, we experimentally approach the tip at a constant current of $11 \mathrm{nA}$. When the extraction bias falls below $90 \mathrm{~V}$, the tip- $\mathrm{CN}$ distance is below $100 \mathrm{~nm}$. We consider that this position corresponds to the $\mathrm{CN}$ apex (i.e., $d=0$ ). Figure 10 shows an experimental voltage evolution during approach. The solid line represents the fitted curve using Eqs. (1) and (6). The fitting parameters are $R_{\mathrm{tip}}=5.5 \mu \mathrm{m}, l_{\mathrm{CNT}}=5.5 \mu \mathrm{m}$, and $r_{\mathrm{CNT}}=15 \mathrm{~nm}$ (coherent with SEM observation of each element).

\section{I-V characterizations}

After the approach, the tip is retracted at a defined position from the CN (between 2 and $10 \mu \mathrm{m}$, typically $4 \mu \mathrm{m}$ ). Several I-V curves are then performed by successively

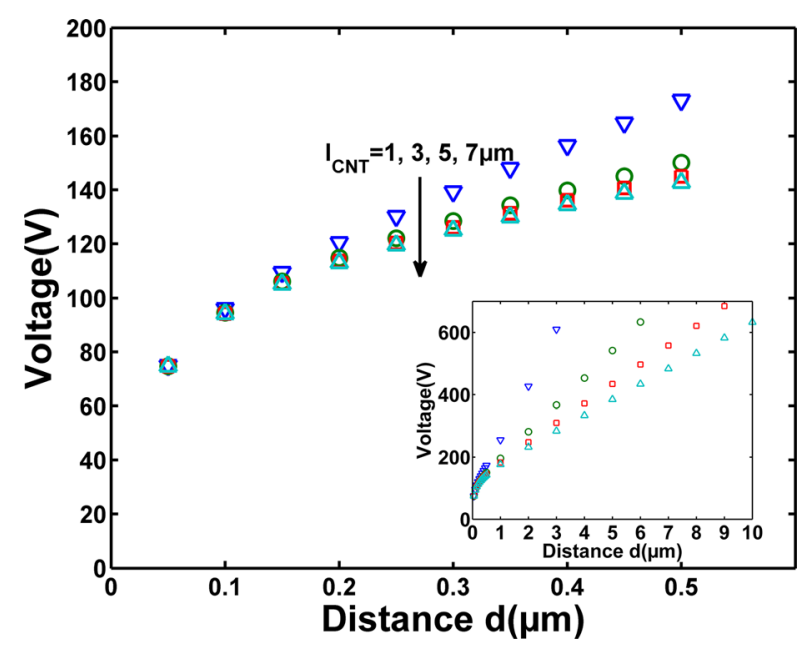

FIG. 9. (Color online) Tip approach simulation for several CN lengths at distances from 50 to $500 \mathrm{~nm}$. Inset: from $50 \mathrm{~nm}$ to $10 \mu \mathrm{m}$.

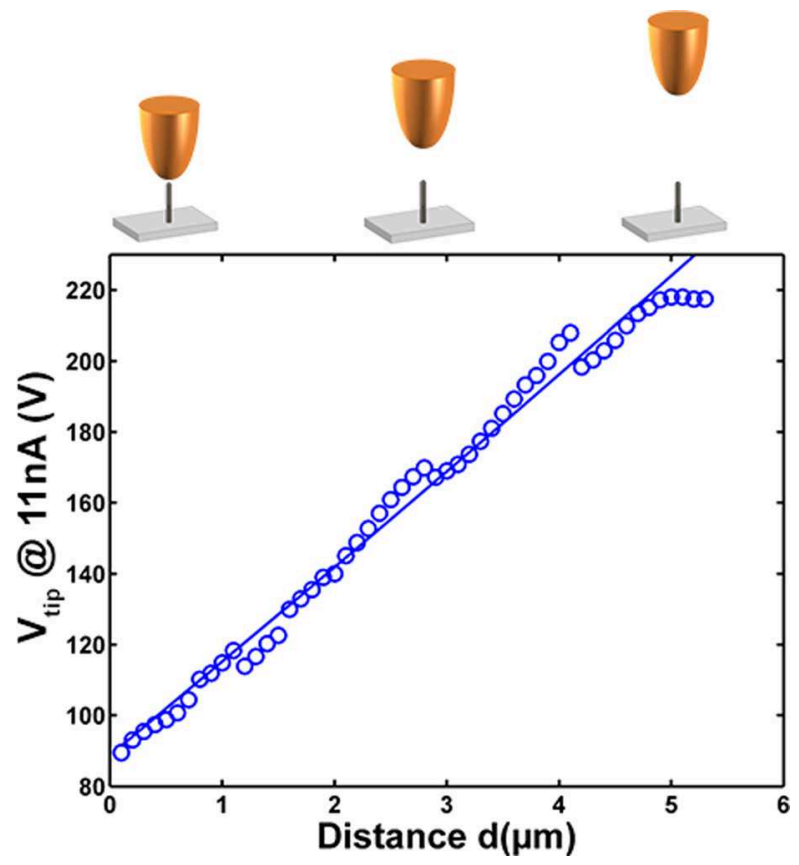

FIG. 10. (Color online) Typical CN approach at a constant current of $11 \mathrm{nA}$.

increasing the voltage and the current compliance in order to gradually increase the current emitted by the $\mathrm{CN}$. The measurement is stopped after the $\mathrm{CN}$ breakdown, from where the maximum current before failure $i_{\max }$ is determined. A typical series of I-V curves of a $\mathrm{CN}$ are shown in Fig. 11.

\section{Determination of the $\mathrm{CN}$ length}

From the length of a reference $\mathrm{CN} l_{\mathrm{CNT}}(1)$ measured by SEM and the position $z(1)$ of the apex of this CN determined in the SAFEM, we directly compute the length of the other $\mathrm{CN} l_{\mathrm{CNT}}(i)$ using the vertical position $z(i)$ of the apex (Fig. 12)

$$
l_{\mathrm{CNT}}(i)=l_{\mathrm{CNT}}(1)+(z(i)-z(1)) .
$$

In order to evaluate the accuracy of our measurement, we determined the length of $19 \mathrm{CNs}$ by SEM and compared these values to the lengths determined by the approach procedure (see Sec. III B 2). A good agreement between the length measured with the SAFEM and the one observed by SEM has been obtained (Fig. 13). The standard deviation of the difference between these two lengths was amounted to $0.4 \mu \mathrm{m}$.

\section{Determination of the field enhancement factor}

The anode tip radius of curvature, the length of each $\mathrm{CN}$, and the working distance being known, we compute the $\gamma_{R \text { tip }}\left(l_{\mathrm{CNT}}, H\right)$ factor for each CN using Eq. (5).

Figure 11 shows that $\mathrm{I}-\mathrm{V}$ curves are reproducible up to the $\mathrm{CN}$ breakdown. From the measured I-V characteristics, the slopes $S$ of the Fowler-Nordheim plots are then determined in the linear low current regime. Hence, we can calculate the enhancement factor using Eq. (9). Since several I-V 

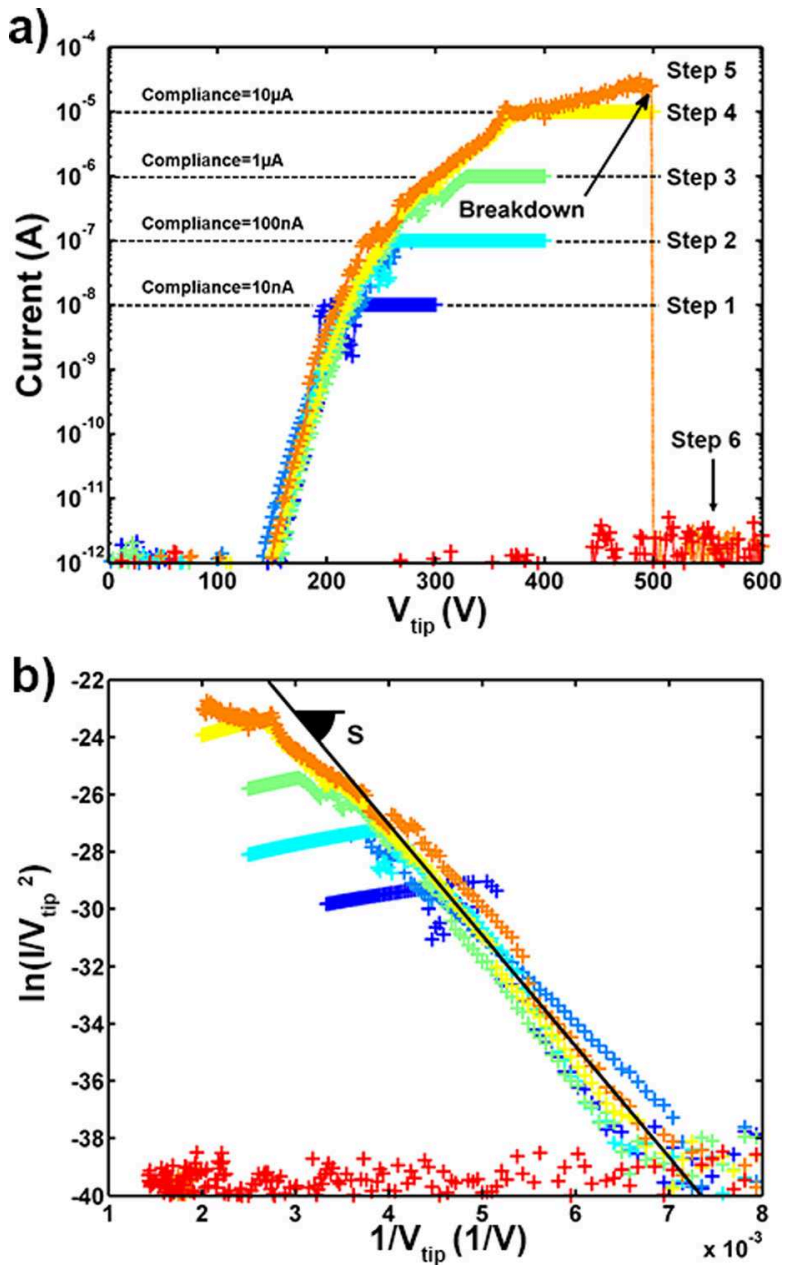

FIG. 11. (Color online) (a) Typical series of $\mathrm{I}\left(\mathrm{V}_{\text {tip }}\right)$ curves and (b) Fowler-Nordheim curves of a CN. During the step 1 (respectively, 2, 3, and 4 ), the current compliance is kept at $10 \mathrm{nA}$ (respectively, $100 \mathrm{nA}, 1 \mu \mathrm{A}$, and $10 \mu \mathrm{A})$. Note that when current compliance is reached, tip voltage is limited.

curves are performed, we use the mean of the enhancement factors calculated until $\mathrm{CN}$ breakdown.

\section{RESULTS AND DISCUSSION}

\section{A. Determination of the absolute field enhancement factor}

We performed I-V characterizations on a single $\mathrm{CN}$ at working distances between $100 \mathrm{~nm}$ and $9 \mu \mathrm{m}$. By extracting the slope of each Fowler-Nordheim curve, we calculated its enhancement factor using our analytical expression at each distance (Fig. 14). On one hand, using our model, an almost constant enhancement factor of $240 \pm 20$ is obtained even if

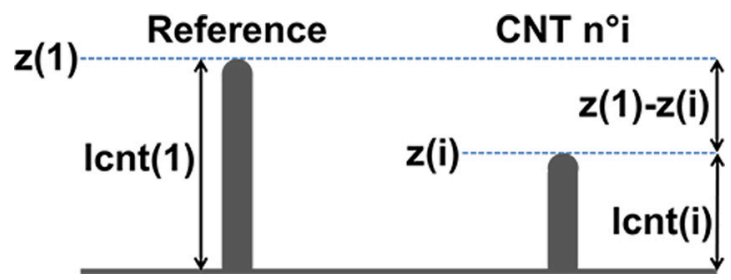

FIG. 12. (Color online) Determination of the length of the CNs.

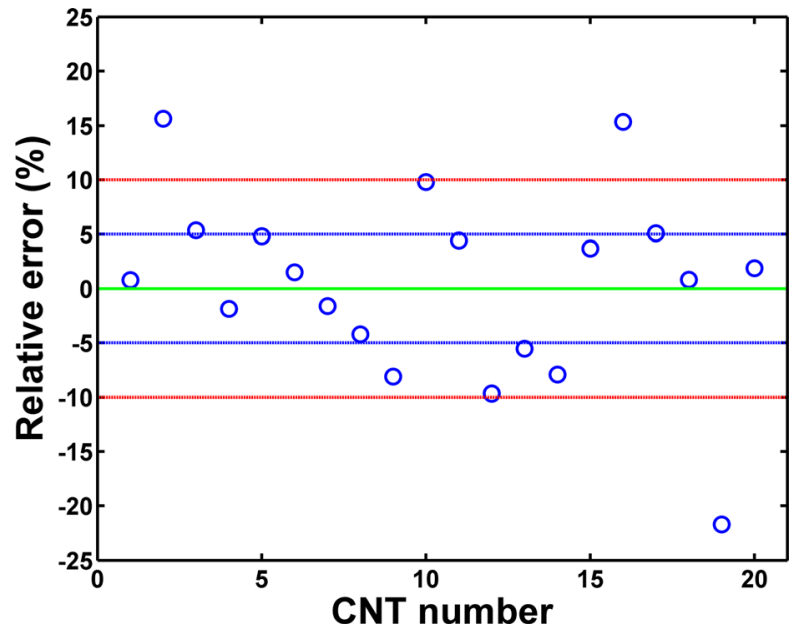

FIG. 13. (Color online) Length measurement relative error on 19 CNs.

$d$ varies from $100 \mathrm{~nm}$ to $9 \mu \mathrm{m}$. On the other hand, the use of the simple "parallel plate" expression for the gap field leads to enhancement factors that strongly depend on $d$.

On the same sample under investigation, we performed the procedure described previously on $100 \mathrm{CNs}$ at three different working distances $(2,4$, and $8 \mu \mathrm{m})$. Figures 15 (a) and 15(b) show, respectively, the enhancement factors calculated with classical approach, i.e., the parallel plate model, and with our novel model. Using the classical approach, the distributions depend on the working distance. Using our new method, the three distributions are identical [Fig. 15(b)]. This validates the proposed model and experimental procedure to quantitatively determine the field enhancement factor of CNs using the SAFEM.

\section{B. Enhancement factor versus emitter length correlation}

$\mathrm{CN}$ models ${ }^{20-22}$ generally express the enhancement factor as a quasilinear function of the length assuming a constant apex radius. Here, we have direct experimental access to these two parameters, i.e., the enhancement factor and the

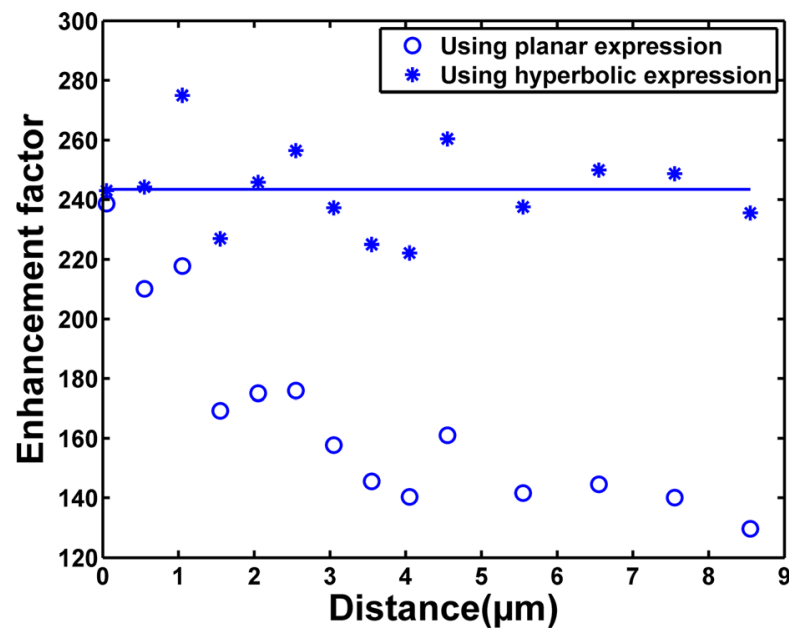

Fig. 14. (Color online) Enhancement factor evolution with the working distance from $100 \mathrm{~nm}$ to $9 \mu \mathrm{m}$. 

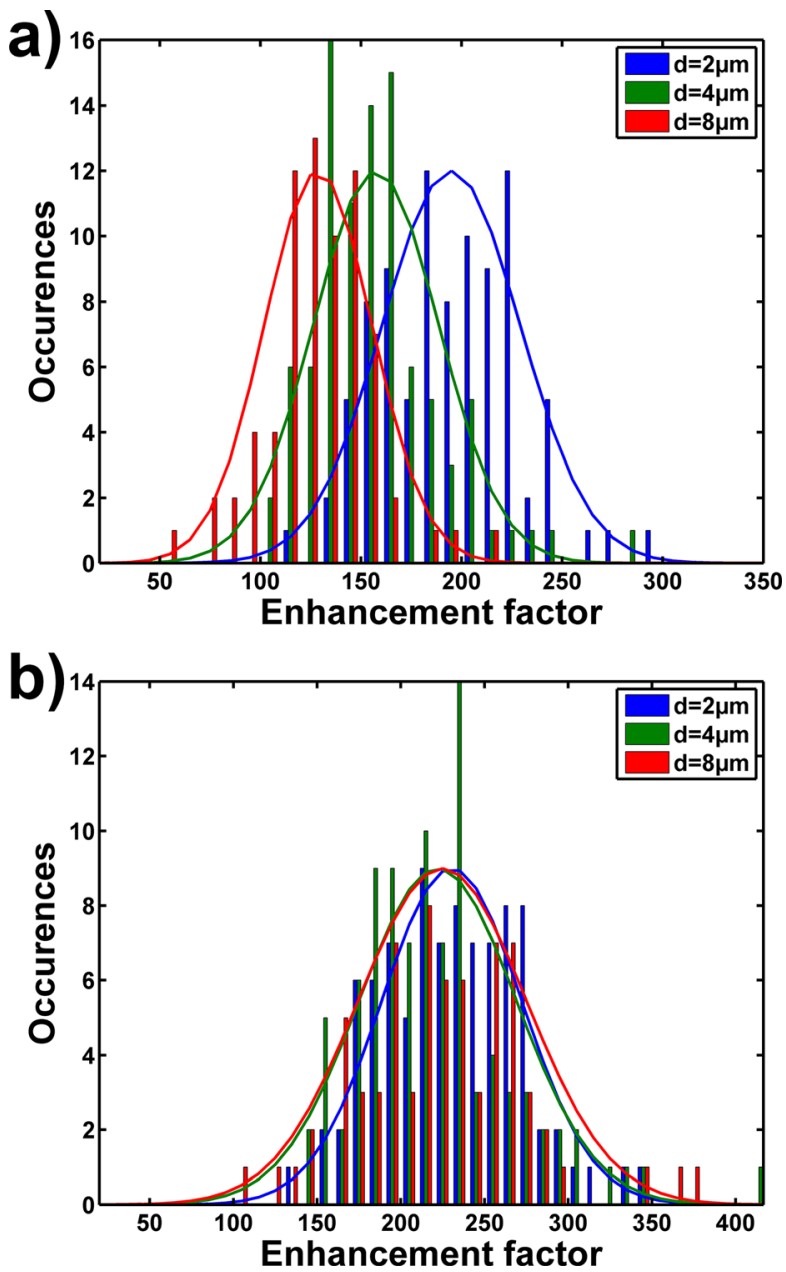

FIG. 15. (Color online) (a) Enhancement factor distributions calculated with the simple parallel plate expression. (b) Enhancement factor distributions calculated with our novel method.

emitter height, of a large set of $\mathrm{CN}$ emitters. As illustrated in Fig. 16, a strong linear correlation between these two parameters is observed, confirming the models and suggesting a rather small variation of the apex radius. The slope $S_{r}$ of the

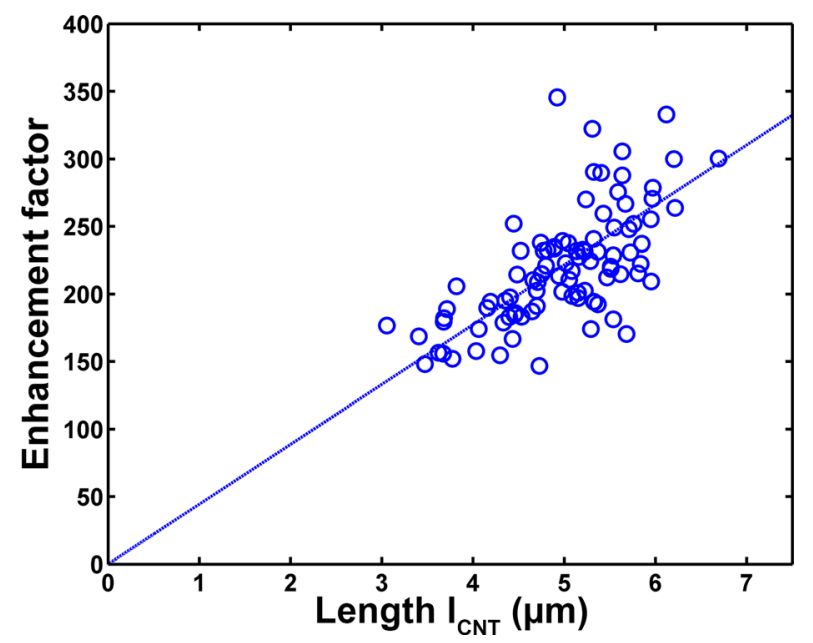

FIG. 16. (Color online) Enhancement factor vs emitter length. The solid line is the linear fit. The slope of this curve is $S_{r}=4.410^{7} \mathrm{~m}^{-1}$.
TABLE II. CN maximum currents at three working distances.

\begin{tabular}{lccc}
\hline \hline Distance $d(\mu \mathrm{m})$ & 2 & 4 & 8 \\
Mean value $(\mu \mathrm{A})$ & 12 & 12 & 14 \\
Median value $(\mu \mathrm{A})$ & 10 & 10 & 13 \\
Standard deviation $(\mu \mathrm{A})$ & 8 & 8 & 8 \\
\hline \hline
\end{tabular}

fitted curve is equal to $4.4 \times 10^{7} \mathrm{~m}^{-1}$. This slope gives a mean effective radius of the $\mathrm{CN}$ population equal to $0.7 / S_{r}=16 \mathrm{~nm}$. This value is coherent with the CN morphology observed by SEM. The deviation around the fitted curve can be explained by the $\mathrm{CN}$ radii distribution.

\section{Maximum current distribution}

Our experimental measurement procedure also provides the maximum current before failure emitted by each $\mathrm{CN}$, since the I-V measurements are performed until breakdown. The mean and median maximum current and the standard deviation of these distributions are summarized in Table II. The values are fairly independent of the anode tip to $\mathrm{CN}$ distance, revealing that the measured breakdown currents are intrinsic to $\mathrm{CN}$ and do not depend on external factor (ion bombardment, voltage dependent arcing, etc.).

A possible correlation between the maximum current and the enhancement factor can be analyzed. Figure 17 shows the maximum current as a function of enhancement factor. Even if the largest maximum currents have been obtained with the CNs exhibiting the lowest enhancement factors, no clear correlation can be extracted. According to models developed by Bonard and Klinke ${ }^{26}$ and Vincent et al., ${ }^{27}$ experimental measurements on the same CN (Refs. 26 and 27) have shown a correlation between the maximum current and the $\mathrm{CN}$ aspect ratio (or enhancement factor). In this studied $\mathrm{CN}$ array, the strong dispersion of the maximum current cannot only be explained by the dispersion of $\mathrm{CN}$ length. The results can be explained by a large dispersion of the electrical contact between $\mathrm{CNs}$ and the substrate or by a large
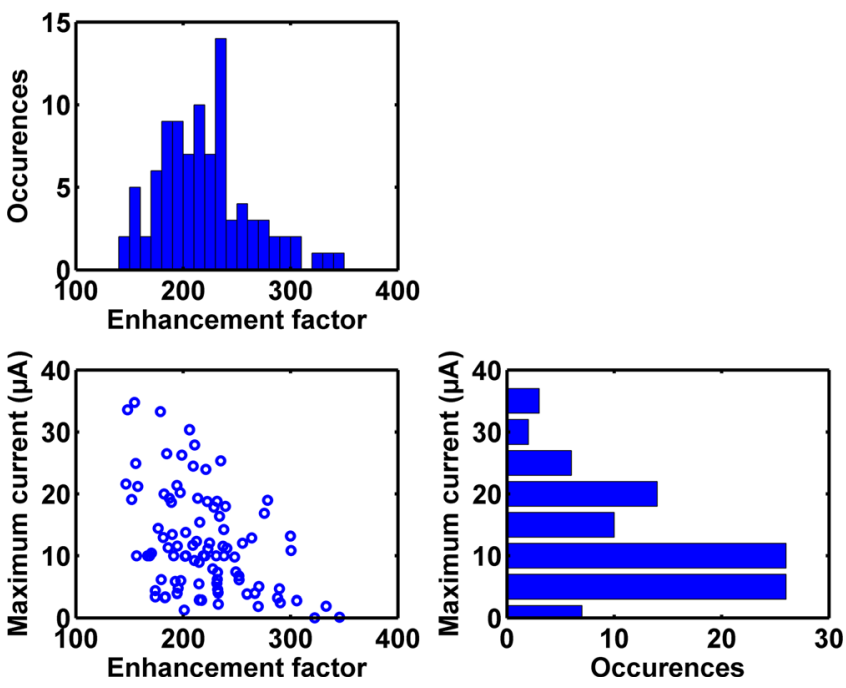

FIG. 17. (Color online) Maximum current before failure $(\mu \mathrm{A})$ vs enhancement factor and their corresponding distributions. 
dispersion of crystalline quality of the CNs constituting the array. In fact, the electrical and thermal conductivities of the CNs depend strongly on their crystalline quality. ${ }^{28,29}$

\section{SUMMARY AND CONCLUSIONS}

The field emission properties of a large number of individual and vertically aligned CNs have been studied using a scanning field emission microscope. In previous studies using similar tip- $\mathrm{CN}$ configurations, it was assumed that the electric field between the tip and the $\mathrm{CN}$ was uniform. In this case, the extracted enhancement factor depends on the tip- $\mathrm{CN}$ distance and thus the exact value cannot be determined. We developed a new analytical model and experimental procedure to precisely measure their enhancement factor. The calculation of the electric field at $\mathrm{CN}$ apex is performed by determining the local potential between the anode-tip and the substrate. Finite elements simulations confirmed the analytical model. Experimental results showed that the use of this model and procedure allows to quantitatively determine the field enhancement factor produced by a $\mathrm{CN}$. We have measured the length, the enhancement factor, and the maximum current before failure of 300 individual CNs. According to proposed models, we experimentally observed a good correlation between the enhancement factor and the $\mathrm{CN}$ length. The correlation between the maximum current and the enhancement factor has been studied. Unlike experiments performed on the same $\mathrm{CN}$ (using shortening steps), we did not observe a clear dependence between enhancement factors and maximum currents as modeled and measured by Vincent $e t$ al. ${ }^{27}$ In the studied array, the strong dispersion of the maximum current cannot only be explained by the dispersion of $\mathrm{CN}$ length. Among other possibilities, we can infer that these results can be explained by a large dispersion of the electrical contact between the CNs and the substrate or by a large dispersion of the crystalline quality of the CNs constituting the array. Indeed, the electrical and thermal conductivities of the CNs depend strongly on their crystalline quality. Our methodology, in particular, for maximum current extraction, coupled to transmission electron microscopy, to infer for crystalline quality, could be a way to estimate the correlation of these two parameters.

SAFEM and the developed model and procedure reveal to be essential regarding optimization of field emitters on both critical parameters that are enhancement factors distributions and maximum current distributions. The determination of these two parameters is essential to understand and optimize the field emission properties from a $\mathrm{CN}$ array.
Indeed, the statistical parameters found by SAFEM measurements can be used for large cathodes simulations and the evaluation of their performances. These optimizations will lead to high current density cathodes for new generation of high performances TWT and x-ray sources.

${ }^{1}$ W. B. Choi et al., Appl. Phys. Lett. 75, 3129 (1999).

${ }^{2}$ D. R. Whaley, R. Duggal, C. M. Armstrong, C. L. Bellew, C. E. Holland, and C. A. Spindt, IEEE Trans. Electron Devices 56, 896 (2009).

${ }^{3}$ J. Zhang, G. Yang, Y. Cheng, B. Gao, Q. Qiu, Y. Z. Lee, J. P. Lu, and O. Zhou, Appl. Phys Lett. 86, 184104 (2005).

${ }^{4}$ C. A. Spindt, I. Brodie, L. Humphrey, and E. R. Westerberg, J. Appl. Phys. 47, 5248 (1976).

${ }^{5}$ W. P. Kang, J. L. Davidson, M. Howell, B. Bhuva, D. L. Kinser, D. V. Kerns, Q. Li, and J. F. Xu, J. Vac. Technol., B 14, 2068 (1996).

${ }^{6}$ F. C. K. Au, K. W. Wong, Y. H. Tang, Y. F. Zhang, I. Bello, and S. T. Lee, Appl. Phys. Lett. 75, 1700 (1999).

${ }^{7}$ C. J. Lee, T. J. Lee, S. C. Lyu, Y. Zhang, H. Ruh, and H. J. Lee, Appl. Phys. Lett. 81, 3648 (2002).

${ }^{8}$ K. B. K. Teo et al., Nature 437, 968 (2005).

${ }^{9}$ E. Minoux et al., Nano Lett. 5, 2135 (2005).

${ }^{10}$ S. T. Purcell, P. Vincent, C. Journet, and V. T. Binh, Phys. Rev. Lett. 88, 105502 (2002).

${ }^{11}$ R. H. Fowler and L. Nordheim, Proc. R. Soc. London A 119, 173 (1928).

${ }^{12}$ K. B. K. Teo, M. Chhowalla, G. A. J. Amaratunga, W. I. Milne, D. G. Hasko, G. Pirio, P. Legagneux, F. Wyczisk, and D. Pribat, Appl. Phys. Lett. 79, 1534 (2001).

${ }^{13}$ L. Nilsson, O. Groening, C. Emmenegger, O. Kuettel, E. Schaller, L. Schlapbach, H. Kind, J.-M. Bonard, and K. Kern, Appl. Phys. Lett. 76, 2071 (2000).

${ }^{14}$ J.-M. Bonard, K. A. Dean, B. F. Coll, and C. Klinke, Phys. Rev. Lett. 89, 197602 (2002).

${ }^{15}$ R. E. Burgess, H. Kroemer, and J. M. Houston, Phys. Rev. 90, 515 (1953).

${ }^{16}$ R. Gao, Z. Pan, and Z. L. Wang, Appl. Phys. Lett. 78, 1757 (2001).

${ }^{17}$ N. de Jonge, M. Allioux, M. Doytcheva, M. Kaiser, K. B. K. Teo, R. G. Lacerda, and W. I. Milne, Appl. Phys. Lett. 85, 1607 (2004).

${ }^{18}$ O. Gröning, O. M. Küttel, Ch. Emmenegger, P. Gröning, and L. Schlapbach, J. Vac. Sci. Technol., B 18, 665 (2000).

${ }^{19}$ J.-M. Bonard, N. Weiss, H. Kind, T. Stöckli, L. Forró, K. Kern, and A. Châtelain, Adv Mater. 13, 184 (2001).

${ }^{20}$ C. J. Edgecombe and U. Valdrè, J. Microsc. 203, 188 (2001).

${ }^{21}$ G. C. Kokkorakis, A. Modinos, and J. P. Xanthakis, J. Appl. Phys. 91, 4580 (2002).

${ }^{22}$ X. Q. Wang, M. Wang, P. M. He, Y. B. Xu, and Z. H. Li, J. Appl. Phys. 96, 6752 (2004).

${ }^{23}$ L. Nilsson, O. Groening, O. Kuettel, P. Groening, and L. Schlapbach, J. Vac. Sci. Technol., B 20, 326 (2002).

${ }^{24}$ K.-F. Hii, R. R. Vallance, S. B. Chikkamaranahalli, M. P. Mengüç, and A. M. Rao, J. Vac. Sci. Technol., B 24, 1081 (2006).

${ }^{25}$ J. Lekner, Eur. J. Phys. 27, 87 (2006).

${ }^{26}$ J.-M. Bonard and C. Klinke, Phys. Rev. B 67, 115406 (2003).

${ }^{27}$ P. Vincent, S. T. Purcell, C. Journet, and V. T. Binh, Phys. Rev. B 66, 075406 (2002).

${ }^{28}$ C. Yu, S. Saha, J. Zhou, L. Shi, A. M. Cassell, B. A. Cruden, Q. Ngo, and J. Li, J. Heat Transfer 128, 234 (2006).

${ }^{29}$ R. Jin, Z. X. Zhou, D. Mandrus, I. N. Ivanov, G. Eres, J. Y. Howe, A. A. Puretzky, and D. B. Geohegan, Physica B 388, 326 (2007). 